\title{
GCU
}

Glasgow Caledonian

University

University for the Common Good

\section{Basal Tear Osmolarity as a metric to estimate body hydration and dry eye severity}

Wiltshire, C.; Bron, A.J.; Gaffney, E.A.; Pearce, E.Ian

Published in:

Progress in Retinal and Eye Research

DOI:

10.1016/j.preteyeres.2018.02.001

Publication date:

2018

Document Version

Author accepted manuscript

Link to publication in ResearchOnline

Citation for published version (Harvard):

Wiltshire, C, Bron, AJ, Gaffney, EA \& Pearce, El 2018, 'Basal Tear Osmolarity as a metric to estimate body hydration and dry eye severity', Progress in Retinal and Eye Research, vol. 64, pp. 56-64.

https://doi.org/10.1016/j.preteyeres.2018.02.001

\section{General rights}

Copyright and moral rights for the publications made accessible in the public portal are retained by the authors and/or other copyright owners and it is a condition of accessing publications that users recognise and abide by the legal requirements associated with these rights.

Take down policy

If you believe that this document breaches copyright please view our takedown policy at https://edshare.gcu.ac.uk/id/eprint/5179 for details of how to contact us. 
Basal Tear Osmolarity

1

2 TITLE: Basal Tear Osmolarity as a Metric to Estimate Body Hydration and Dry Eye

3 Severity.

4

5

6

$7 \quad$ Short title: Basal Tear Osmolarity

8

9

10

11

12

13

14

15

16

17

18

19

20

21

22

23

24

25

26

27

28

29

30

31 Funding: This research did not receive any specific grant from funding agencies in the public,

32 commercial, or not-for-profit sectors.

33

34 


\begin{tabular}{|c|c|}
\hline & Table of Contents \\
\hline & Abstract \\
\hline 1 & Introduction \\
\hline 2 & Lacrimal Secretion \\
\hline 3 & Tear Osmolarity / Osmolality \\
\hline 3.1 & Diurnal variation of tear osmolarity \\
\hline 4 & Dry Eye Disease \\
\hline 5 & Body Hydration and Dehydration \\
\hline 5.1 & Body Hydration and Tear Osmolarity \\
\hline 6 & Hypothesis \\
\hline 6.1 & $\begin{array}{l}\text { Basal Tear Osmolarity as a Metric in Dry Eye Diagnosis and in the } \\
\text { Estimation of Body Hydration }\end{array}$ \\
\hline 7 & Measurement of tear osmolarity after eye closure \\
\hline 7.1 & Estimating the necessary period of eye closure \\
\hline 7.2 & Measurement of tear osmolarity in conditions of high humidity \\
\hline 8 & $\begin{array}{l}\text { Piloting the effects of eye closure and exposure to high humidity on } \\
\text { tear osmolarity }\end{array}$ \\
\hline 9 & Predicted utility of the BTO in estimating body hydration. \\
\hline \multirow[t]{2}{*}{10} & Summary and Conclusions \\
\hline & References \\
\hline
\end{tabular}


Abstract:

The osmolarities of various bodily fluids, including tears, saliva and urine, have been used as indices of plasma osmolality, a measure of body hydration, while tear osmolarity is used routinely in dry eye diagnosis, the degree of tear hyperosmolarity providing an index of

53 disease severity. Systemic dehydration, due to inadequate water intake or excessive water

54 loss is common in the elderly population, has a high morbidity and may cause loss of life. Its diagnosis is often overlooked and there is a need to develop a simple, bedside test to detect dehydration in this population. We hypothesize that, in the absence of tear evaporation and with continued secretion, mixing and drainage of tears, tear osmolarity falls to a basal level that is closer to that of the plasma than that of a tear sample taken in open eye conditions. We term this value the Basal Tear Osmolarity (BTO) and propose that it may be measured in tear samples immediately after a period of evaporative suppression. This value will be particular to an individual and since plasma osmolarity is controlled within narrow limits, it is predicted that it will be stable and have a small variance. It is proposed that the BTO, measured immediately after a defined period of eye closure, can provide a new metric in the diagnosis of systemic dehydration and a yardstick against which to gauge the severity of dry eye 65 disease.

Key Words: tears, plasma osmolarity, osmolality, systemic dehydration, dry eye 
In this paper we hypothesize that in the healthy eye, tear osmolarity measured after a period of evaporative suppression, represents a basal level of osmolarity close to that of the plasma. It is proposed that such a metric can provide a valuable measure of body hydration and a baseline against which to gauge the severity of dry eye disease (DED).

The aqueous tears occupy the conjunctival sac when the eyes are closed and are redistributed between the fornical and preocular compartments when the eyes open (Gaffney et al., 2010). The preocular compartment splits into two during the upstroke of the blink to form the preocular tear film and the tear menisci, and these are surfaced anteriorly by the tear film lipid layer, which retards evaporation (McDonald and Brubaker, 1971; Peng et al., 2014; Cerretani and Radke, 2014). Once formed, the tear film remains 'perched' throughout the blink interval (Miller, Polse and Radke, 2002) while the menisci provide a conduit for the drainage of tears into the nasolacrimal system (Doane, 1981). The tear film is further divided into the precorneal and prebulbar films.

The aqueous tears derive chiefly as an active secretion of the lacrimal gland (Mircheff, 1989; Turpie et al., 2009; Dartt 2004, 2009; Hodges and Dartt, 2016; Stevenson, Pugazhendhi, and Wang 2016), and to a lesser extent from, the conjunctival epithelium, including the goblet cells (Shiue et al., 2000; Dartt, 2002; Li et al., 2001; Dartt, 2009) and the corneal epithelium

104 (Klyce and Crosson 1985). The size of these additional contributions is not established in 105 humans but in the rabbit it has been calculated that the conjunctival fluid could account for the volume of the basal tear secretion (Shiue et al., 2000; Li et al., 2001). Cerretani and

107 Radke, in their model of human tear dynamics concluded that the contribution of 108 osmotically-induced water flow to the total tear supply, through the conjunctiva and cornea, 109 was in the region of $10 \%$ (Cerretani and Radke, 2014). In patients who have undergone 110 daryoadenectomy (removal of the main and palpebral parts of the lacrimal gland) in the 111 treatment of epiphora (Taiara and Smith, 1973; Hornblass, Guberina, and Herschorn, 1988)

112 or lacrimal gland neoplasia (Rose and Wright, 1992), a proportion of patients fail to develop

113 dry eye and may show no reduction in the Schirmer response, implying an adequate supply of 114 tear fluid from some source other than the main and palpebral lacrimal gland (Stevenson 115 Pugazhendhi and Wang, 2016). This source could include the accessory lacrimal glands and 
the conjunctival and corneal epithelia but such reports do permit the relative contribution of these sites to residual tear secretion to be determined. The accessory glands account for about a tenth of the total lacrimal mass (Allansmith et al., 1976). Thus they do not shed light on the normal contribution of the ocular surface epithelia to tear production and this fraction remains unknown in humans. The lacrimal component increases substantially during emotional tearing and in the reflex response to intense light or a corneal foreign body (Murube, 2009; Dartt, 2002; Nelson and Wright, 1986).

\section{LACRIMAL SECRETION}

The acinar cells of the lacrimal gland represent about $80 \%$ of the glandular mass while the duct cells represent 10-12\% (Dartt, 2002). The lacrimal secretion, derived from the lacrimal acini, is modified as it passes through the lacrimal ducts and its composition differs from that of the lacrimal fluid that is delivered into the conjunctival sac. Regulated secretion of the major acinar proteins, lysozyme, lactoferrin, lipocalin, and peroxidase, involves exocytosis, a rapid process involving the fusion of acinar apical membranes with those of the apical secretory vesicles, occurring in response to an appropriate stimulus. The duct epithelium modifies the primary lacrimal secretion by the addition of water and electrolytes, particularly of $\mathrm{K}^{+}$and $\mathrm{Cl}^{-}$ions (Dartt, Moller, and Poulsen, 1981; Mircheff, 1989; Ubels et al., 1994; Dartt, 2009; Katona et al., 2014). In the rabbit, it has been estimated that the duct cells secrete about $30 \%$ of the lacrimal fluid (Katona et al., 2014) but the figure for human lacrimal fluid is not known.

The lacrimal, conjunctival and corneal fluids are mixed and distributed by blinking (Gaffney et al., 2010) and to a lesser extent by eye movements (Yokoi, Bron and Georgiev, 2014) and it is this composite fluid that is termed the tears and is assayed in meniscus samples.

\section{TEAR OSMOLARITY / TEAR OSMOLALITY}

The osmolarity of a solution is the number of osmoles per litre of solvent, usually expressed as milliosmoles. The osmolality of a solution is the number of osmoles per kilogram of solution. In the literature related to systemic disease and plasma, osmolality is the preferred term while in the tear literature the term osmolarity is more often used. Where an estimate for serum is made from the concentration of selected serum constituents, the value is usually 


\section{Basal Tear Osmolarity}

150

151

152

153

154 For the tears, based on a meta-analysis of several studies using depression of freezing point, 155

156

157

158

159

160

161

162

163

164

165

166

167

168

169

170

171

172 When the eyes are open, the osmolarity of the tears is modified by evaporation, to an extent

173

174

175

176

177

178

179

180

expressed as osmolarity. Clinically the numerical difference between the two terms may be small and but the formula selected to make the calculation is of importance (Hooper, 2015a). Here we use either term, according to its literature source. or vapour pressure measurement, tear osmolarity (tOsm) has been reported to be $302 \pm 9.7$ $\mathrm{mOsm} / \mathrm{L}$ in normal adults (Tomlinson et al., 2006). Similar values were reported by Sullivan et al. (Sullivan et al., 2010) - 302.2 $8.3(\mathrm{n}=75)$, Jacobi et al. (Jacobi et al., 2011) - 301 mOsm/L ( $\mathrm{n}=95)$, Keech et al. (Keech et al., 2013) - $301.2 \pm 7.2 \mathrm{mOsm} / \mathrm{L}$ ( $\mathrm{n}=15)$, Eldridge, et al (Eldridge et al., 2010) - 301.8 $\pm 10.5 \mathrm{mOsms} / \mathrm{L}$ and by Li et al. (Li et al., 2012) $298.0 \pm 14.2 \mathrm{mOsms} / \mathrm{L}$, based on smaller subsets. In all these studies, tear osmolarity values were obtained using the TearLab ${ }^{\circledR}$ device, which depends on the measurement of electrical impedance and therefore the presence of charged particles in solution and therefore, in the tears, mainly ions and to a much lesser extent, proteins. The presence of urea and of glucose in the tears is not registered by this device.

Tear hyperosmolarity is the central mechanism of dry eye acting in part directly on epithelial cells (Kam et al., 2016) and in part by generating downstream inflammatory events at the ocular surface (Bron et al., 2017). Tear film break-up in the blink interval amplifies tear hyperosmolarity and additionally, degrades optical performance when tear instability and breakup intrude upon the visual axis (Chao et al., 2016).

that depends on ambient humidity (Madden, Tomlinson and Simmons, 2013; Lee et al., 1999), air temperature (Abusharha Pearce and Fagehi, 2015) and airflow (Peng et al., 2014), the size of the palpebral aperture and the length of the blink interval, which determines the period of evaporation (Tsubota and Nakamori, 1995; Tsubota, 1998). Tear osmolarity is increased by a low relative humidity $(\mathrm{RH})$, high wind speed, raised air temperature, a wide palpebral aperture and an extended blink interval (Chao et al., 2016). It is generally stated that the lacrimal fluid is secreted as an iso-osmotic, or slightly hypo-osmotic fluid (Terry and Hill 1978; Gilbard and Farris, 1979; Niimi et al., 2013) compared with plasma. Tears 


\section{Basal Tear Osmolarity}

181

182

183

184

185

186

187

188

189

190

191

193

194

195

196

197

198

199

200

201

202

203

204

205

sampled from the menisci are considered to have a higher level of osmolarity than that of secreted tears, (Mishima and Maurice, 1961; Mishima, 1965; Niimi et al., 2013; Cerretani and Radke, 2014) and that of the tear film, the latter due to the differential effect of evaporation on these two compartments during the blink interval (Gaffney et al., 2010). The ionic composition of the tears is determined by the secretory process (Dartt, 2009; Mircheff, 1989; Katona et al., 2014) and it has been noted that the relative proportions of electrolytes measured in tear fluid and plasma differ (van Haeringen, 1981). Thus, while the concentrations of $\mathrm{Na}^{+}$and $\mathrm{HCO}^{-}$in human tears are close to those of the plasma (Krogh Lund and Pedersen-Bjergaard, 1945; Hind and Goyan, 1949; Thaysen and Thorn, 1954; Yoshimura and Hosokawa, 1963), those of $\mathrm{K}^{+}$and $\mathrm{Cl}^{-}$are higher in the tears (Rismondo et al., 1989), and there is evidence in the rabbit (Mircheff, 1989; Ubels et al.,1994) and rat (Ubels et al., 2006) that $\mathrm{K}^{+}$and $\mathrm{Cl}^{-}$ions are added to the lacrimal fluid by the epithelial cells of the lacrimal duct. In a carefully designed study in rabbits, reported by Yoshimura and Hosokawa (Yoshimura and Hosokawa, 1963) in which tear osmolarity was measured by freezing point depression, tear osmolarity was $17 \mathrm{mOsm} / \mathrm{L}$ higher in the tears than in plasma (329 in tears versus $312 \mathrm{mOsm} / \mathrm{L}$ in plasma) due to the higher $\mathrm{K}^{+}$and $\mathrm{Cl}^{-}$ion concentrations in the tears. In other reports, also in the rabbit, lacrimal fluid osmolarity was reported to be inversely proportional to flow rate, with hyperosmolarity encountered at low secretory rates (Bothelo and Martinez, 1973; Gilbard and Dartt, 1982). Botelho and Martinez postulated that at low flow rates, water might be reabsorbed in the ducts, distal to the acini. If this situation applies to human tears then it cannot be excluded that human lacrimal fluid too, is slightly hyperosmolar with respect to plasma.

\subsection{Diurnal Variation of Tear Osmolarity}


206 Various researchers have reported a diurnal variation of tOsm, with the tears found to be

207 hypo-osmotic on waking (Terry and Hill, 1978; Niimi et al., 2013). Niimi et al. (Niimi, et al.,

208 2013) used a TearLab® apparatus modified to register lower levels of osmolarity, to study

209 the relationship between diurnal variations of tear osmolarity, central corneal thickness and

210 corneal deswelling over the day. The TearLab® device measures tear osmolarity on the basis

211 of electrical impedance and has the advantage that measurement is made directly on the

212 sampled fluid, without risk of evaporative loss. The authors recorded osmolarity at bedtime

213 (base-line), upon waking after 6-8.5 hours sleep and at intervals after waking. Tears on

214 waking were found to be significantly hypo-osmotic (264 $\pm 14 \mathrm{mOsms} / \mathrm{L})$ compared with the

215 pre-sleep, baseline values of $297 \pm 15 \mathrm{mOsms} / \mathrm{L}$ and those encountered later in the day. Tear

216 osmolarity rose quickly in the first 10 minutes after waking, reaching baseline levels within

217 the first 40 minutes $(\mathrm{P}=0.085)$. These authors attributed the hypo-osmolarity of tears on

218 waking to the suppression of evaporation by lid closure and possibly to reflex tearing

219 occurring on eye opening. Also, their subjects were instructed to blink 3 times and to squeeze

220 their eyes shut to release fresh tears prior to tear collection, and this may have influenced the

221 outcome. Given that the level of osmolarity reported fell below that normally cited for plasma

222 osmolarity, (i.e. 285-295 mOm/kg (Matz, 1996; Stookey, 2005; Cheuvront et al., 2010) reflex

223 tearing at the time of sampling may have contributed to the low value, but does not explain it.

225 In conditions of high tear flow, such as with reflex tearing, tOsm falls from that recorded in

226 unstimulated, open eye conditions. In a study of six subjects with normal eyes, exposed to the

227 beam of the slit lamp for five seconds, to induce reflex tearing, tOsm measured by a

228 depression of freezing point method, fell from $303.2 \pm 7.2 \mathrm{mOsm} / \mathrm{kg}$ (range 287-312

$229 \mathrm{mOsm} / \mathrm{kg}$ ), to $289.5 \pm 6.8 \mathrm{mos} / \mathrm{kg}$ (range $275-298 \mathrm{mos} / \mathrm{kg}$ ), a 5\% decrease, which was

230 statistically significant $(\mathrm{p}<.001)$ (Nelson and Wright, 1986).

\section{DRY EYE DISEASE}

234 Dry eye disease is a symptomatic eye disorder in which drying of the exposed ocular surface 235 by evaporative water loss, results in tear hyperosmolarity. This damages the ocular surface,

236 either directly or by a chain of events causing inflammatory ocular surface damage. (Bron et

237 al., 2017) There are two major subtypes. In aqueous-deficient dry eye (ADDE), tear

238 hyperosmolarity is due to evaporation from a reduced tear flow, caused by a reduction in

239 lacrimal secretion. In evaporative dry eye (EDE), tear hyperosmolarity arises from an 
excessive evaporative loss, caused by a failure of the barrier function of the tear film lipid

241 layer and amplified by tear film break up. Tear hyperosmolarity has been proposed as the

242 best single diagnostic test of dry eye (Korb, 2000). In a multicentre study the most sensitive

243 threshold distinguishing normal from mild/moderate dry eye disease was $308 \mathrm{mOsm} / \mathrm{L}$ and

244 the most specific cut off was $315 \mathrm{mOsm} / \mathrm{L}$ (Lemp et al., 2011). In terms of tear osmolarity,

245 severity is compared with values in subjects with normal eyes. The hypothesis that we

246 present below gives the opportunity to use a tOsm value obtained in the same individual,

247 rather than derived from a normal, control population.

\section{BODY HYDRATION and DEHYDRATION}

Total body water (TBW) makes up about $50 \%-60 \%$ of the body mass, with about two thirds being intracellular, predominantly in lean tissue, and the remainder extracellular (Danziger and Zeidel, 2015). Blood contributes about 8\% to the TBW (Rikkert, 1998; Bossingham, et vapour, urine and faeces and is replaced by fluid intake and by water contained in foodstuffs. At sea level, the amount of water lost as respiratory vapour is balanced by metabolic water

257 production (Cheuvront et al., 2014).

Regulation of water balance is fundamental to survival and is achieved by a combination of water conservation (renal) and acquisition (thirst). Water conservation results from the action of arginine vasopressin (AVP or antidiuretic hormone) on renal water absorption (Baron, 2015). AVP is synthesised in the supraoptic and paraventricular nuclei of the hypothalamus and delivered to the posterior pituitary, from which it is released (Bourque, 2008) in response to signals from hypothalamic osmoreceptors (eg.TRPV1) (Ciura, 2006; Leng, 1982). These, acting as membrane stretch-receptors, signal changes in cell volume (Liedtke, 2000) in response to changes in plasma osmolality (pOsm). A rise in pOsm creates an osmotic gradient through which the effects of water loss are shared between the intracellular fluid (ICF) and extracellular fluid (ECF) compartments (Cheuvront and Kenefick, 2014). An increase in neuronal firing stimulates the release of AVP from the posterior pituitary, resulting in renal water reabsorption, urinary concentration and water conservation

271 (Cheuvront et al., 2013). A rise is pOsm also stimulates an increase in water intake in 272 response to thirst (Egan, et al., 2003) which is independent of the action of AVP and results 273 from direct neural signaling (Denton, et al., 1999; Bourque, 2008). Peripheral osmoreceptors, 


\section{Basal Tear Osmolarity}

274

275

276

277

278

279

280

281

282

283

284

285

286

287

288

289

290

291

292

293

294

295

296

297

298

299

300

301

302

303

304

305

306

307

eg. in the gut, also play a role (Bourque, 2008).

The osmoreceptor neurons in the hypothalamus are believed to encode an osmotic set-point (Bourque, 2008) that keeps pOsm from deviating by more than $1-2 \%$ in an individual (Bourque, 2008; Cheuvront and Kenefick, 2014). The pOsm set point for AVP release is lower than that which stimulates thirst by $10 \mathrm{mmol} / \mathrm{kg}$ or more (Cheuvront, et al., 2013).

In this way, in normally hydrated subjects, hydration is maintained within narrow limits. (Danziger and Zeidel, 2015). For plasma, this is between 285-295 mOsm/kg. (Matz, 1996; Stookey, 2005; Cheuvront, 2010). Thomas et al. cite a broader range for serum osmolality of 275 to $<295 \mathrm{mOsmol} / \mathrm{kg}$, (Thomas et al., 2008) but $<2 \%$ of free-living people have a pOsm $<285 \mathrm{mOsmol} / \mathrm{kg}$ when they consume $\geq 3.0 \mathrm{~L}$ fluid per day (Stookey, 2005).

Clinical dehydration has been defined as a loss of body water, with or without salt, at a rate greater than the body can replace it (Thomas et al., 2008). This article is concerned with the water-loss dehydration, which is accompanied by intracellular dehydration, plasma hyperosmolarity and, usually, plasma hypernatraemia. It is also termed hypohydration, hyperosmotic hypovolaemia and dehydration with minimal salt loss (Cheuvront and Kenefick, 2014). Water-loss dehydration may also be due to hyperglycaemia, in which case it is accompanied by hyponatraemia. Extracellular dehydration, caused by a loss of iso-osmotic body fluids, as in secretory diarrhea, involves a reduction in ECF water and will not be discussed here. (Cheuvront and Kenefick, 2014),

Plasma or serum osmolality, measured directly, or estimated from the chemical composition of these fluids (Hooper, 2015a; 2016) has long been used as a clinical index of body hydration (Armstrong, 2007; Cheuvront et al., 2010; Baron et al., 2015) serving as the gold standard against which other less invasive methods are compared in the diagnosis of dehydration. Clinical or 'current' dehydration is defined by a plasma osmolality of $>300$ $\mathrm{mOsm} / \mathrm{kg}$ and preclinical, or 'impending' dehydration by a plasma osmolality of $>295$ and $\leq$ $300 \mathrm{mOsm} / \mathrm{kg}$. Impending dehydration can be managed by a planned adjustment of an individual's daily fluid intake, whereas current dehydration demands urgent water replacement to prevent life-threatening complications. Loss of body mass $\geq 3 \%$, signifying loss of TBW, recorded over a period of 7 days, is also used as a reference standard in the detection of dehydration, (Hooper et al., 2016). 
Basal Tear Osmolarity

308

309 The frequency of current dehydration in the elderly population is high, with impending

310 dehydration reported as $40 \%$ in those aged 70-90 years, in the US NHANES III cohort, with

311 a further $28 \%$ exhibiting current dehydration (referred to in this report as, 'overt

312 hypertonicity', $\geq 300 \mathrm{mmol} / \mathrm{L}$ ) (Stookey, 2005). Consequently, dehydration, contributing to

313 the risk of chronic diseases such as urolithiasis, hypertension and coronary heart disease,

314 (Xiao, Barber, and Campbell, 2004), is a leading cause of hospitalization and death in the

315 elderly. (Manz and Wentz, 2005; Oei et al., 2016) A number of factors contribute to this.

316 Older people have a smaller body fluid reserve than younger people, due to reduced muscle

317 volume (Rickert et al.,1997; Martin et al., 1994) and lose more intracellular water and less

318 interstitial fluid in response to heat and exercise (Morgan, et al., 2002). Food intake and the

319 number of episodes of drinking decrease with age (Gaspar, 1999) and the elderly fail to drink

320 adequate amounts of fluid in response to dehydration (Rolls and Phillips, 1990) in part due to

321 a decreased sense of thirst (de Castro, 1992). The urinary concentrating ability of the kidney

322 also declines with age (Davies et al., 1995; Lindeman et al., 1985; Morely, 2000; Sands,

323 2012; Hooper et al., 2016) and, additionally, an increased use of diuretics or laxatives in older

324 people contributes to greater fluid loss (Mentes, 2006). Other, cognitive and physical factors,

325 reduce fluid intake (Lindeman et al., 2000; Zizza et al., 2009) and drinking may be restricted

326 deliberately as a measure to control incontinence (Hooper et al., 2016; He et al., 2015). Those

327 with dementia may forget to drink, as daily routines are lost and social contacts diminish

328 (Hooper et.al., 2016).

329

330 The risk of dehydration is increased in elderly patients in long-term care. Hooper et al.

331 (Hooper et al., 2016) reported a frequency of $20 \%$ in a population of care home residents

$332(\mathrm{n}=188)$ with a mean age 86 years, with renal, cognitive and diabetic status consistently

333 associated with the risk of dehydration. Wolff et al. (Wolff et al., 2015) in another UK study,

334 basing the diagnosis of dehydration on the presence of hypernatraemia on admission to

335 hospital (plasma $\mathrm{Na}>145 \mathrm{mmol} / \mathrm{L}$ ), found a 5-fold increase in the occurrence of dehydration

336 in patients admitted to hospital from care homes (adjusted odds ratio [AOR]: 5.32, 95\% CI:

337 3.85-7.37), compared to that in patients admitted from home, and roughly a two-fold greater

338 risk of in-hospital death (AOR: 1.97, 95\% CI: 1.59-2.45) (Wolff et al., 2015).

340 This background emphasizes the need to detect dehydration in the elderly, both in the wider

341 community and in individuals in care (Hydration for Health Initiative, 2012). Dehydration is 


\section{Basal Tear Osmolarity}

less likely to be overlooked in the hospital population, where serum osmolarity can be readily calculated from blood samples. While it is generally agreed that the estimation of plasma osmolality or serum osmolarity, provide the best single assessment of body hydration (Hooper et al., 2016; Thomas et al., 2008) such tests are not routinely performed in the community or in primary or residential care settings (Leibovitz, 2007). Assessment by health or social care workers is more likely to be based on the demonstration of reduced thirst, sense of a dry mouth, furrowing of the tongue, loss of skin turgor, a dry axilla, slow capillary refilling after compression of the nailbed, and increase in urine colour, which appear to be poor indicators of dehydration in older adults (Hooper et al., 2016). More formal measurements, of urinary specific gravity, or of salivary or urinary osmolarity, or bioimpedance have also been used. In a systematic review of tests validated to detect current water-loss dehydration in older people, Hooper et al (Hooper et al., 2015b) found that only three stand-alone tests showed any ability to diagnose water-loss dehydration, as indicated by a serum osmolality $\geq 295 \mathrm{mOsm} / \mathrm{kg}$, with a sensitivity $\geq 0.60$ and specificity $\geq 0.75$. These were, missing drinks between meals, expressing fatigue and, in some reports, bioimpedance (BIA) at $50 \mathrm{kHz}$. No tests were clearly useful in diagnosing current water-loss dehydration (serum osmolality $>300 \mathrm{mOsm} / \mathrm{kg}$ ).

This report (Hooper et al., 2015b) and that of the earlier, US Panel on Dietary Reference Intakes, (Panel on Dietary Reference Intakes, 2004) emphasize the need to develop a valid, simple and non-invasive screening test of dehydration in the community, to enable the identification and management of water loss dehydration in older adults.

\subsection{Body Hydration and Tear Osmolarity.}

Although lacrimal secretion is influenced by vascular filtration pressure (Botelho et al., 1976) it is the active, energy-requiring, secretory process that determines the final composition of the tears and hence its osmolarity (Dartt Moller and Poulsen, 1981; Mircheff, 1989). Tear osmolarity is also influenced by plasma osmolarity and the extent to which this occurs in humans has been demonstrated by Walsh and colleagues (Fortes et al., 2011; Walsh Fortes, and Esmaeelpour 2011; Walsh et al., 2012) who reported a positive relationship between whole body hydration measured as pOsm, and tOsm, in subjects exposed to systemic dehydration (Fortes et al., 2011). In a study conducted in an environmental chamber, a group of young adults in their $20 \mathrm{~s}$, was exposed to systemic dehydration, equivalent to 2 to $3 \%$ loss of body mass, generated by a combination of water-deprivation and a period of physical 
exercise. Tear osmolarity followed pOsm closely during the evolution of dehydration and, like pOsm, was restored to normal during rehydration. In this study, the pre-exercise pOsm was $288 \pm 5 \mathrm{mOsm} / \mathrm{kg}$. In two trials, the mean tOsm correlated strongly with mean pOsm at each time point $(r=0.93, \mathrm{P}<0.001)$, suggesting that tOsm could serve as a minimally invasive surrogate for body hydration. Fortes et al. reported a sensitivity of $80 \%$ and specificity of $92 \%$ using tOsm to detect systemic dehydration (Fortes et al., 2011). In a subsequent study, the authors reported that pOsm may be raised in patients with dry eye disease with the implication that the raised tOsm could be a consequence of body dehydration (Walsh Fortes and Esmaeelpour, 2011). In a subsequent letter they expressed the view that this could lead to a misdiagnosis of dry eye in patients who suffered from systemic dehydration, (Walsh et al., 2012) but Tomlinson et al. (Tomlinson Madden and Pearce, 2011) in response, pointed out that the persistent presence of a tear hyperosmolarity within the range consistent with the diagnosis of DED, in conjunction with supportive clinical features, would imply the actual presence of DED. Importantly, as noted by Walsh et al. (Walsh Fortes and Esmaeelpour, 2011), since the risk of both dry eye (Uchino et al., 2006; Moss Klein and Klein, 2008; Guo et al., 2010) and systemic dehydration (Cheuvront and Kenefick, 2014), increases with age, the value of a raised tOsm in the diagnosis of systemic dehydration is the elderly will be reduced (Walsh Fortes and Esmaeelpour, 2011; Walsh et al., 2012; Tomlinson Madden and Pearce, 2011).

It is evident that the occurrence of tear hyperosmolarity due to DED is a potential source of false positives when using tear osmolarity to diagnose systemic dehydration, when based on the results of random, open eye tear samples. However, if, as we propose below, the tOsm measurement were to be made after a period of evaporative suppression, this difficulty would be overcome and a realistic estimate of both body hydration status and of dry eye severity could be achieved

\section{HYPOTHESIS}

\subsection{Basal Tear Osmolarity as a Metric in Dry Eye Diagnosis and in the Estimation of}

\section{Body Hydration}

As noted, tear hyperosmolarity is the central mechanism in dry eye disease. At present, for diagnostic purposes, when a patient is suspected of having dry eyes, their tear osmolarity, derived from a meniscus sample, is compared with population norms obtained from subjects 
410

411

412

413

414

415

416

418

419

420

421

422

423

424

425

426

427

428

429

430

431

432

433

434

435

436

437

438

439

440

441

442

443

over a wide age range. It would be more valuable if a comparison could be made with that individual's own tear osmolarity obtained before the onset of dry eye. It is our contention that this value can be obtained in any subject, regardless of the presence or absence of dry eye, simply by subjecting the subject to a period of evaporative suppression prior to tear sampling.

We hypothesize that, in the absence of tear evaporation, with continued lacrimal and conjunctival secretion and adequate tear mixing and drainage, the osmolarity of the tears, equilibrating with the interstitial fluid across the ocular surface epithelia, will fall to a basal levels close to that of the plasma. We consider that this basal value will serve both as a measure of body hydration and as a stable baseline against which to compare the tear hyperosmolarity in dry eye. Tear evaporation is readily prevented by eye closure and we postulate that eye closure for a suitable length of time will drive down tear osmolarity to this basal level, regardless of the osmolar starting point in open eye conditions and therefore regardless of the presence of dry eye. This new metric, which we term the Basal Tear Osmolarity (BTO) will be particular to an individual and is predicted to have a smaller variance than tear meniscus values measured in non-standardised, open eye, conditions and to be relatively uninfluenced by ambient environmental conditions. It is proposed as a potential tool in the diagnosis of systemic dehydration and as a yardstick against which to gauge the severity of dry eye disease.

We propose here that the BTO can obtained by measuring tear osmolarity after a period of eye closure or exposure of the subject to a humid environment in open eye conditions. These approaches are described briefly here:

\section{MEASUREMENT OF TEAR OSMOLARITY AFTER EYE CLOSURE}

In order to explore the effect of lid closure on tear osmolarity it is necessary to estimate the likely period of lid closure required to drive down tear osmolarity to a stable, BTO value. Certain considerations need to be addressed. The hypothesis assumes that with the eyes closed for a suitable period of time, the body of tear fluid contained within the conjunctival sac will be completely replaced by lacrimal fluid, supplemented by a smaller amount of fluid of conjunctival origin and less still of corneal origin. The completeness of this process will depend on tear turnover, mixing and drainage. In the absence of blinking, a deficiency of tear mixing during eye closure might be overcome to a limited extent by performing periodic eye 
444 movements. In patients with ADDE, lacrimal secretion and turnover is, by definition,

445 reduced and it would be predicted that in such individuals, the reduction in tear turnover

446 might increase the time required to drive down tOsm to the BTO value. However, the longer

447 the period of eye closure, the greater the opportunity, for tear fluid in the conjunctival sac to

448 equilibrate with the plasma across the conjunctival epithelium and extracellular space. This

449 equilibration can be predicted to be faster in dry eye disease, since epithelial permeability is

450 increased (Yokoi et al., 1997).

451

452 7.1. Estimating the Necessary Period of Eye Closure

454 On eyelid closure, the elevated tear osmolarity inherited from the open eye will be reduced 455 by cessation of evaporation, by tear turnover and by equilibration across the conjunctival 456 epithelium. The time scale of the former is readily estimated. If the total tear turnover rate is 457 16\% per minute, (Tomlinson Doane and McFadyen, 2009) then the flush-out time is 458 approximately $100 / 16 \mathrm{~min}=6.25 \mathrm{~min}$.

460 Across the surface epithelium, the osmolarity of the tears would lie somewhere between that 461 of the lacrimal fluid and the epithelial fluids. Here, we make a rough estimate of the time 462 taken for the osmolarity of the lacrimal fluid to approximate to that of the conjunctival fluid, 463 considering equilibration across the vascular conjunctiva alone, since the surface area of the 464 human conjunctiva is an order of magnitude greater than that of the cornea (Watsky Jablonski 465 and Edelhauser, 1988).

467 Some idea of the equilibration rate can be approximated from the short circuit current across

468 the epithelium. Using rabbit data, based on the unilateral removal of chloride from either side 469 of a rabbit conjunctival preparation, the change in the short circuit current is on the scale of $4703 \mu \mathrm{Acm}^{-2}$ (Kompella Kim and Lee, 1993). This can be converted into an equilibration rate 471 across the conjunctiva, first dividing by Faraday's constant, $F$, to rewrite the short circuit 472 current in terms of ionic flux. Multiplying by conjunctival surface area (human: $A_{c}=18 \mathrm{~cm}^{2}$ ) 473 (Watsky Jablonski and Edelhauser 1988) converts this flux into a rate of change of total 474 amount of ion. Dividing by tear volume ( $\mathrm{V}=7 \mu \mathrm{l})$ (Tomlinson Doane and McFadyen, 2009), 475 gives the rate of change of concentration. Finally, dividing by a representative, initial 476 concentration difference of chloride across the epithelium in these experiments, taking the 477 value to be around $\mathrm{c}^{*}=100 \mathrm{mM} / 1$ this entails an equilibration rate of 
Basal Tear Osmolarity

478

479

480

481

482

483

484

485

486

487

488

489

490

491

492

493

494

495

496

497

498

499

500

501

502

503

504

505

506

507

508

509

510

511

$$
k=3 \mu \mathrm{Acm}^{-2} \cdot A_{c}\left[F V c^{*}\right]=1.2 \mathrm{e}-3 \mathrm{~s}^{-1} .
$$

The associated equilibration timescale is given by $1 / k \approx 830 \mathrm{~s} \approx 14 \mathrm{mins}$. One must accept the caveat that this is a rough approximation.

In summary, the timescales of the system are such that there will be a relatively rapid wash out of the combined fluids over about 6-7 minutes in the normal eye. If lacrimal fluid hyperosmolarity were to be present, a further equilibration across the conjunctival epithelium will be active on a timescale of, very roughly, 14 minutes. The period of 45 minutes of eye closure adopted in the experiments described below, should therefore be adequate to achieve equilibration. Given its limited surface area, the impact of the less permeable cornea is anticipated to be sub-dominant. These estimates would be modified by variations in tear flow rate and the increase in epithelial permeability encountered in dry eye disease. For comparison, Zhu and Chauhan in a model simulation to determine the impact of moisture chambers on dry eye sufferers, explored the effect of raising the evaporation rate to four times the normal rate and then reducing it back to normal (Zhu and Chauhan, 2007). On the basis of this they predicted a restoration of tear osmolarity to baseline values in about 13 minutes.

\subsection{Measurement of Tear Osmolarity in Open Eye Conditions in High Ambient}

\section{Humidity}

Exposure of a subject whose eyes are open, to an ambient RH of $100 \%$ will also result in a complete suppression of tear evaporation and offers an alternative approach to the estimation of the BTO. Although the value obtained with either approach should be similar there is a practical value in adopting lid closure for clinical purposes, since it does not require a controlled environment chamber or goggles constructed to create a humid environment.

08 However, exposure to a humid environment offers experimental advantages in tracking the downward path of tear osmolarity over time, since meniscus sampling can be conducted at any point throughout the exposure period. Similarly, this open eye approach offers the opportunity to study osmolar recovery on transfer to a non-humid environment. In the study 
512 of Niimi et al. (Niimi et al., 2013) tOsm rose quickly over the first 10 minutes after waking,

513 reaching baseline levels within the first 40 minutes. In high humidity studies it is likely that

514 the fall in osmolarity towards the BTO will be faster than in closed eye conditions, because

515 mixing and drainage will be facilitated by spontaneous blinking, whereas in the closed eye

516 state, mixing will be more restricted.

8. PILOTING THE EFFECTS OF EYE CLOSURE AND EXPOSURE TO HIGH HUMIDITY ON TEAR OSMOLARITY

521 We have performed a preliminary study to estimate the BTO in eight normal subjects and

522 eight dry eye patients, after periods of evaporative suppression achieved by either eye closure

523 or exposure to high relative humidity (Willshire et al., 2017). In the eye closure studies,

524 closure was maintained for a period of 45 minutes, and eye movements were performed from

525 time to time to achieve some degree of tear mixing. In a separate study, subjects were

526 exposed to an atmosphere of 70\% RH and tOsm was measured in both eyes, every 15

527 minutes, for a period 45 minutes. Studies were preceded by measurement of tOsm outside

528 the controlled environment chamber (CEC), in uncontrolled, clinic conditions, to provide

529 baseline values. Tear osmolarity was significantly reduced after eye closure, in the right and

530 left eyes analysed independently, in both normal subjects and dry eye patients, to levels in

531 the range accepted for plasma osmolality, i.e. between 285-295 mOsm/L. The average

532 tOsm measured in the left eye of 8 normal subjects, prior to eye closure, was $293.1 \pm$

$5335.54 \mathrm{mOsm} / \mathrm{L}$ and was $285.9 \pm 5.54 \mathrm{mOsm} / \mathrm{L}(\mathrm{p}=0.006)$ immediately after eye opening.

534 Corresponding values in 8 patients with mild DED, were $302.3 \pm 12.4 \mathrm{mOsm} / \mathrm{L}$ in the

535 clinic, falling to $286.1 \pm 6.60 \mathrm{mOsm} / \mathrm{L}$ following eye closure $(\mathrm{p}=0.01)$ (Figure 1$)$.

536 Similar results, also statistically significant, were demonstrated in the right eye

537 (Willshire et al., 2017). When these subjects were exposed to 70\% RH, which was not

538 expected to suppress evaporation completely, a significant fall in tOsm occurred in one eye

539 only in the normal group, but not in the dry eye group.

9. PREDICTED UTILITY OF THE BTO IN ESTIMATING BODY HYDRATION

543 Our hypothesis predicts that total evaporative suppression will drive down tear osmolarity to

544 the BTO in both normal subjects and in patients with DED. The BTO value obtained will be 
545 dependent on that individual's hydration state and as noted, would be expected to be confined

546 within narrow limits, reflecting the tight control of plasma osmolarity. This gets over the

547 difficulty that a raised tOsm measured in open eye conditions cannot distinguish the effect of

548 suboptimal body hydration from that of DED (Walsh Fortes and Esmaeelpour 2011; Walsh et

549 al., 2012) and eliminate concerns that environmental factors such as desiccation, sun, wind or

550 rain and behavioural factors such as outdoor exercise, (causing movement convection), that

551 might act as measurement confounders, limiting the application of this approach within

552 sports, wilderness and military medicine (Sollanek et al., 2012; Cheuvront and Kenefick,

553 2014).

554

555 In normal subjects the difference between the BTO, measured as proposed here and a random

556 meniscus reading measured in clinic conditions, may be predicted to be small, however, in

557 DED, the difference should rise progressively with increasing disease severity. We propose

558 that this differential will provide a better index of dry eye severity in an individual patient

559 than would be afforded by a comparison with a control population.

560

\section{SUMMARY AND CONCLUSIONS}

562

563 The BTO is proposed here as a new metric for the diagnosis of systemic dehydration and as a

564 yardstick against which to gauge the severity of dry eye disease. This could meet the need

565 expressed by several authors for a technology that is simple, rapid and non-invasive

566 (Armstrong, 2005; Institute of Medicine, 2005; Sollanek, et al., 2012; Ungaro et al., 2015;

567 Holland et al., 2017). Such a metric could be of utility in several ways.

569 1. It is anticipated that the BTO will provide a better diagnostic surrogate for whole body

570 (plasma) hydration than tear osmolarity measured under non-standardised ambient

571 conditions. As a minimally invasive, point-of-care diagnostic test that can be deployed at the

572 bedside, it may be of value in the diagnosis of dehydration in the elderly. However, if it

573 transpires from future studies, that the BTO can be acquired after a short period of eye

574 closure, say 15 minutes or less, regardless of the starting level of tOsm, then the utility of the

575 test will be greatly enhanced and it may be of value in other situations where individuals are

576 exposed to excessive water loss or deprivation, as in sports and the military environment.

577 Ungaro et al. (2015) compared mean tOsm (averaged between right and left eyes) with pOsm

578 in a group of male athletes, before and after exercise tasks conducted on a stationary cycle 
579

580

581

582

583

584

585

586

587

588

589

590

591

592

593

594

595

596

597

598

599

600

601

602

603

604

605

606

607

608

609

610

611 3. While the difference between the BTO and the level of tear hypermolarity are conceived to

612 be a measure of dry eye severity, it also indicates the fold increase in tear osmolarity due to

ergometer. These were carried out under controlled environmental conditions, with or without water restriction leading to up to $3 \%$ of body mass loss, and also after rehydration. They found that tOsm tracked group changes in hydration status similarly to pOsm but that individual responses of tOsm were less predictable. They concluded that tOsm is a valid indicator of hydration status at the group level, but that large differences among subjects in the response of tOsm to changes in hydration status limited its validity at the individual level (Ungaro et al., 2015). A similar conclusion was drawn in another study conducted under field conditions involving a self-paced $10 \mathrm{~km}$ run, in which participants were exposed to varied conditions of temperature, humidity and wind speed (Holland et al., 2017). In that study, although significant reductions in body mass and increases in plasma osmolality, tear osmolarity and urine specific gravity were observed, the pre- to post-exercise change in tear osmolarity was not significantly correlated with plasma osmolality, relative body mass loss, or urine osmolality or specific gravity. It may be surmised that exclusion of environmental exposure, as proposed for a closed-eye BTO test, might have revealed a correlation between tOsm and pOsm in such studies. Importantly, since sampling is performed immediately after a period of eye closure, it will not be influenced by ambient environment or the presence of dry eye disease; the tOsm will be driven down to the BTO level in any individual. The time taken to achieve the BTO value in a closed eye test will be important in determining its practicality, particularly under field conditions.

2. It is proposed that measurement of the BTO will be of value in assessing the severity of dry eye, since it will indicate how far tear osmolarity has risen above the basal level in that individual. The set point of pOsm about which pOsm oscillates during the maintainance of osmolar hydration differs between individuals and the threshold and slope (sensitivity) of the AVP response to pOsm change, is under genetic control (Zerbe et al., 1999; Cheuvront et al., 2013). Also, in treating patients with dry eye and trying to restore a normal tear osmolarity, the BTO will provide an appropriate reference point against which to judge successful treatment. Experimentally, the approach also offers the opportunity to explore the time taken for tear osmolarity to return to DED levels on eye opening, in defined ambient conditions. This has some bearing on the recuperative value afforded by eye closure during sleep and may differ between the main subtypes of DED. 
613 evaporative water loss and therefore how much of the increased concentration of a given

614 solute reflects the concentrating effect of evaporation and how much is due to increased

615 expression of that molecule. It is important to know this, since the level of tear lacrimal

616 protein falls in ADDE but is predicted not to do so in EDE, where lacrimal function is normal

617 (Bron et al., 2009).

618

619 4. The role of tear evaporation in causing DED has long been recognized (Lemp, 1995;

620 DEWS 2007; DEWS II - Bron et al., 2017) and treatment measures designed to reduce

621 evaporative water loss are part of the therapeutic approach to dry eye disease, either by the

622 provision of moisture-conserving spectacles (Tsubota Yamada and Urayama, 1994) or, in

623 severe DED, by performing tarsorrhaphy, as a temporary measure (Welch and Baum, 1988;

624 Nelson, 1989; Valim et al., 2015). It is self-evident but rarely emphasized, however, that

625 while in the dry eye patient, overnight eye closure during sleep removes the physical basis of

626 hyperosmolarity, interactions with the proinflammatory conditions induced by the closed eye

627 state (Sack et al., 2000) make its therapeutic implications difficult to predict. It is not

628 expected that the effect of eye closure on tOsm and the level of inflammatory mediators in

629 the tears and tissues will be concordant. We predict that while tOsm will fall, the level of

630 inflammatory mediators will not be affected in the short term and might even increase.

631

632 5. In the diagnosis of DED it is recommended that tOsm is measured in both eyes, since the

633 between-eye difference increases in DED and is of diagnostic value. In the report of Lemp et

634 al. (Lemp et al., 2011) normal subjects demonstrated a mean inter-eye difference of $6.9 \pm 5.9$

$635 \mathrm{mOsms} / \mathrm{L}$, whereas patients with mild or moderate DED demonstrated a difference of $11.7 \pm$

$63610.9 \mathrm{mOsms} / \mathrm{L}$ and those with severe DED, a difference of $26.5 \pm 22.7 \mathrm{mOsms} / \mathrm{L}$. It is likely

637 that, for the detection of systemic dehydration, it will be sufficient to take the measurement in

638 one eye only, after bilateral eye closure, although this will need confirmation based on

639 comparative studies.

640

641 6. It has been argued that plasma osmolarity may be of less value in the diagnosis of chronic

642 dehydration than acute dehydration (Armstrong, 2007; Baron et al., 2015), for instance

643 because dehydration may cause a rise in plasma osmolarity that still falls within the normal

644 range and yet represents dehydration in that individual. However, in the environment of a

645 care home for the elderly it would be possible to obtain baseline BTO readings when the

646 patient was in a state of euhydration, against which to compare subsequent measurements. 
648 7. In summary, measurement of the Basal Tear Osmolarity is proposed as a new diagnostic 649 approach worthy of further consideration. Its utility in the diagnosis of body dehydration in

650 the elderly could usefully be studied in the environment of the nursing home and compared to

651 that achieved using current practices. Preliminary studies suggest that, as predicted, the 652 variance of BTO measurements in both normal and DED subjects, is lower than that of the 653 tOsm measured in uncontrolled, clinic conditions, (Willshire et al., 2017). Future studies are 654 planned in larger populations and will include a direct comparison of the BTO with pOsm 655 at different levels of body hydration and the measurement of the BTO in open eye conditions 656 at an RH close to $100 \%$. By conducting such studies in patients with different subtypes of 657 DED, we hope to better define the period of eye closure required for a substantive, clinical 658 BTO test.

659

660 Although current studies have indicated, in a preliminary way, a numerical similarity between BTO values measured by the TearLab ${ }^{\circledR}$ device and reference values for pOsm in normally hydrated individuals, it must be recognised that osmolarity measured by electrical

663 impedance does not fully represent the concentration of all particles in solution and hence 664 must be expected to slightly underestimate the full osmolarity of the tears.

665 The TearLab® device detects the presence of charged particles, such as ions and does not 666 recognize uncharged molecules such as urea or glucose. Urea is a permeant molecule whose 667 concentration in the tears is similar to that in the plasma, accounting, according to one source, 668 for around $6 \mathrm{mOsm} / \mathrm{L}$ in normal subjects (Gavrilov et al., 2000). Tear glucose, in non669 diabetic subjects contributes about $0.2 \mathrm{mOsm} / \mathrm{L}$ (Sen and Sarin, 1980). Thus it may be 670 predicted that when direct comparisons of tOsm and pOsm are made, the pOsm will be about

$6716 \mathrm{mOsm} / \mathrm{L}$ higher than the simultaneously measured tOsm. This prediction needs to be 672 confirmed by a direct comparison of tOsm with pOsm in the same individuals combined with 673 measurements of plasma composition, but does not diminish the potential values of the 674 proposed approach.

678 Acknowledgements: We thank Professor Sir John Grimley Evans and Dr. Sam N Cheuvront 679 for helpful discussions in the preparation of this manuscript. 
681

682

683

684

685

686

687

688

689

690

691

692

693

694

695

696

697

698

699

700

701

702

703

704

705

706

707

708

709

710

711

712

713

714

715

716

717

718

719

720

721

722

723

724

725

726

727

728

729

730

\section{References}

Abusharha, A. A., E. I. Pearce, and R. Fagehi. 2015. 'Effect of Ambient Temperature on the Human Tear Film', Eye Contact Lens.

Allansmith, M. R., et al. (1976). "Plasma cell content of main and accessory lacrimal glands and conjunctiva." Am J Ophthalmol 82(6): 819-826.

Armstrong, L. E. (2005). "Hydration assessment techniques." Nutr Rev 63(6 Pt 2): S40-54.

Armstrong, L. E. 2007. 'Assessing hydration status: the elusive gold standard', J Am Coll Nutr, 26: 575S-84S.

Baron, S., M. Courbebaisse, E. M. Lepicard, and G. Friedlander. 2015. 'Assessment of hydration status in a large population', Br J Nutr, 113: 147-58.

Bourque, C. W. (2008). "Central mechanisms of osmosensation and systemic osmoregulation." Nat Rev Neurosci 9(7): 519-531.

Bossingham, M. J., et al. (2005). "Water balance, hydration status, and fat-free mass hydration in younger and older adults." Am J Clin Nutr 81(6): 1342-1350.

Botelho, S. Y. and E. V. Martinez (1973). "Electrolytes in lacrimal gland fluid and in tears at various flow rates in the rabbit." Am J Physiol 225(3): 606-60

Botelho, S. Y., E. V. Martinez, C. Pholpramool, H. C. Prooyen, J. T. Janssen, and A. De Palau. 1976. 'Modification of stimulated lacrimal gland flow by sympathetic nerve impulses in rabbit', Am J Physiol, 230: 80-4.

Bron, A. J., N. Yokoi, E. Gaffney, and J. M. Tiffany. 2009. 'Predicted phenotypes of dry eye: proposed consequences of its natural history', Ocul Surf, 7: 78-92.

Bron, A. J., et al. (2017). "TFOS DEWS II pathophysiology report." Ocul Surf 15(3): 438510.

Cerretani, C. F., and C. J. Radke. 2014. 'Tear dynamics in healthy and dry eyes', Curr Eye Res, 39: 580-95.

Chao, W., C. Belmonte, J. M. Benitez Del Castillo, A. J. Bron, H. S. Dua, K. K. Nichols, G. D. Novack, S. Schrader, M. D. Willcox, J. S. Wolffsohn, and D. A. Sullivan. 2016. 'Report of the Inaugural Meeting of the TFOS i(2)= initiating innovation Series: Targeting the Unmet Need for Dry Eye Treatment', Ocul Surf, 14: 264-316.

Cheuvront, S. N., B. R. Ely, R. W. Kenefick, and M. N. Sawka. 2010. 'Biological variation and diagnostic accuracy of dehydration assessment markers', Am J Clin Nutr, 92: 56573.

Cheuvront, S. N., et al. (2013). "Physiologic basis for understanding quantitative dehydration assessment." Am J Clin Nutr 97(3): 455-462.

Cheuvront, S. N. and R. W. Kenefick (2014). "Dehydration: physiology, assessment, and performance effects." Compr Physiol 4(1): 257-285.

Ciura, S. and C. W. Bourque (2006). "Transient receptor potential vanilloid 1 is required for intrinsic osmoreception in organum vasculosum lamina terminalis neurons and for normal thirst responses to systemic hyperosmolality." J Neurosci 26(35): 9069-9075.

Danziger, J., M.L. Zeidel Osmotic homeostasis, Clin. J. Am. Soc. Nephrol. CJASN. 10 (2015) 852-862. doi:10.2215/CJN.10741013.

Dartt, D. A. 2002. 'Regulation of mucin and fluid secretion by conjunctival epithelial cells', Prog Retin Eye Res, 21: 555-76. - 2004. 'Dysfunctional neural regulation of lacrimal gland secretion and its role in the pathogenesis of dry eye syndromes', Ocul Surf, 2: 76-91.

- 2009. 'Neural regulation of lacrimal gland secretory processes: relevance in dry eye diseases', Prog Retin Eye Res, 28: 155-77.

Dartt, D. A., M. Moller, and J. H. Poulsen. 1981. 'Lacrimal gland electrolyte and water secretion in the rabbit: localization and role of $(\mathrm{Na}++\mathrm{K}+)$-activated ATPase', $J$ Physiol, 321: 557-69. 
731

732

733

734

735

736

737

738

739

740

741

742

743

744

745

746

747

748

749

750

751

752

753

754

755

756

757

758

759

760

761

762

763

764

765

766

767

768

769

770

771

772

773

774

775

776

777

778

779

780

Davies, I., et al. (1995). "Age-associated alterations in thirst and arginine vasopressin in response to a water or sodium load." Age Ageing 24(2): 151-159.

De Castro, J. M. (1992). "Age-related changes in natural spontaneous fluid ingestion and thirst in humans." J Gerontol 47(5): P321-330.

Denton, D., et al. (1999). "Neuroimaging of genesis and satiation of thirst and an interoceptor-driven theory of origins of primary consciousness." Proc Natl Acad Sci U S A 96(9): 5304-5309.

DEWS 2007 'The definition and classification of dry eye disease: report of the Definition and Classification Subcommittee of the International Dry Eye WorkShop (2007)'. Ocul Surf, 5: 75-92.

Doane, M. G. 1981. 'Blinking and the mechanics of the lacrimal drainage system', Ophthalmology, 88: 844-51.

Egan, G., et al. (2003). "Neural correlates of the emergence of consciousness of thirst." Proc Natl Acad Sci U S A 100(25): 15241-15246.

Eldridge, D.C., B.D. Sullivan, M.D. Berg, M.A. Lemp, and D.S. Durrie. 2010. 'Longitudinal Variability of Tear Film Osmolarity in Normal and Dry Eye Patients', IOVS Abstract 3379, 51.

Fortes, M. B., B. C. Diment, U. Di Felice, A. E. Gunn, J. L. Kendall, M. Esmaeelpour, and N. P. Walsh. 2011. 'Tear fluid osmolarity as a potential marker of hydration status', Med Sci Sports Exerc, 43: 1590-7.

Gaffney, E. A., J. M. Tiffany, N. Yokoi, and A. J. Bron. 2010. 'A mass and solute balance model for tear volume and osmolarity in the normal and the dry eye', Prog Retin Eye Res, 29: 59-78.

Gaspar, P. M. (1999). "Water intake of nursing home residents." J Gerontol Nurs 25(4): 23 29.

Gavrilov, V., et al. (2000). "Tear/Plasma Urea Ratio as a Correction Coefficient for Drug Monitoring in Tears."] Pharm Technol 16: 18-20.

Gilbard, J. P. and R. L. Farris (1979). "Tear osmolarity and ocular surface disease in keratoconjunctivitis sicca." Arch Ophthalmol 97(9): 1642-1646.

Guo, B., P. Lu, X. Chen, W. Zhang, and R. Chen. 2010. 'Prevalence of dry eye disease in Mongolians at high altitude in China: the Henan eye study', Ophthalmic epidemiology, 17: 234-41.

He, S., et al. (2015). "Unmet Need for ADL Assistance Is Associated With Mortality Among Older Adults With Mild Disability." J Gerontol A Biol Sci Med Sci 70(9): 1128-1132.

Hind, H. W., and F. M. Goyan. 1949. 'The hydrogen ion concentration and osmotic properties of lacrimal fluid', J Am Pharm Assoc, 38: 477-9.

Hodges, R. R., and D. A. Dartt. 2016. 'Signaling Pathways of Purinergic Receptors and Their Interactions with Cholinergic and Adrenergic Pathways in the Lacrimal Gland', $J$ Ocul Pharmacol Ther, 32: 490-97.

Holland, J. J., et al. (2017). "Tear osmolarity is sensitive to exercise-induced fluid loss but is not associated with common hydration measures in a field setting." J Sports Sci: 1-8.

Hooper, L., et al. (2015a). "Diagnostic accuracy of calculated serum osmolarity to predict dehydration in older people: adding value to pathology laboratory reports." BMJ Open 5(10): e008846.

Hooper, L., A. Abdelhamid, N.J. Attreed, W.W. Campbell, A.M. Channell, P. Chassagne, K.R. Culp, S.J. Fletcher, M.B. Fortes, N. Fuller, Clinical symptoms, signs and tests for identification of impending and current water - loss dehydration in older people, Cochrane Libr. (2015b). http://libweb.anglia.ac.uk.

Hooper, L., et al. (2016). "Which Frail Older People Are Dehydrated? The UK DRIE Study." J Gerontol A Biol Sci Med Sci 71(10): 1341-1347. 
781

782

783

784

785

786

787

788

789

790

791

792

793

794

795

796

797

798

799

800

801

802

803

804

805

806

807

808

809

810

811

812

813

814

815

816

817

818

819

820

821

822

823

824

825

826

827

828

829
Hornblass, A., C. Guberina, and B. J. Herschorn. 1988. 'Palpebral dacryoadenectomy for epiphora', Ophthal Plast Reconstr Surg, 4: 227-30.

Hydration for Health Initiative. Hydration in the aging: a review of current knowledge. April 2012. Available at: www.h4hinitiative.com/tools

Jacobi, C., A. Jacobi, F.E. Kruse, C. Cursiefen, Tear film osmolarity measurements in dry eye disease using electrical impedance technology, Cornea. 30 (2011) 1289-1292.

doi:10.1097/ICO.0b013e31821de383.

Institute of Medicine, 2005. Water, Dietary Reference Intakes for Water, Sodium, Chloride, Potassium, and Sulfate. . Washington, DC, National Academy Press: 73-185.

Kam, W., et al. (2016). "Does hyperosmolarity induce an irreversible process leading to human corneal epithelial cell death? (abstract) "Invest Ophthalmol Vis Sci 2016b; \#, ARVO Abstract 6161.

Katona, M., E. Vizvari, L. Nemeth, A. Facsko, V. Venglovecz, Z. Rakonczay, Jr., P. Hegyi, and E. Toth-Molnar. 2014. 'Experimental evidence of fluid secretion of rabbit lacrimal gland duct epithelium', Invest Ophthalmol Vis Sci, 55: 4360-7.

Keech, A., et al. (2013). "Impact of time between collection and collection method on human tear fluid osmolarity." Curr Eye Res 38(4): 428-436.

Klyce, S. D., and C. E. Crosson. (1985). 'Transport processes across the rabbit corneal epithelium: a review', Curr Eye Res, 4: 323-31.

Kompella, U. B., K. J. Kim, and V. H. Lee. 1993. 'Active chloride transport in the pigmented rabbit conjunctiva', Curr Eye Res, 12: 1041-8.

Korb, D. R. 2000. 'Survey of preferred tests for diagnosis of the tear film and dry eye', Cornea, 19: 483-6.

Krogh, A., C.G. Lund, and Pedersen-Bjergaard. 1945. 'The osmotic concentration of human lacrymal fluid', Acta physiol. scandinav., 10: 88-90.

Lee, S. C., C. S. Poon, X. D. Li, and F. Luk. 1999. 'Indoor air quality investigation on commercial aircraft', Indoor Air, 9: 180-7.

Leibovitz, A., et al. (2007). "Dehydration among long-term care elderly patients with oropharyngeal dysphagia." Gerontology 53(4): 179-183.

Lemp, M. A., A. J. Bron, C. Baudouin, J. M. Benitez Del Castillo, D. Geffen, J. Tauber, G. N. Foulks, J. S. Pepose, and B. D. Sullivan. 2011. 'Tear osmolarity in the diagnosis and management of dry eye disease', Am J Ophthalmol, 151: 792-98 e1.

Lemp, M.A. . 1995. 'Report of the National Eye Institute/Industry workshop on Clinical Trials in Dry Eyes. ', CLAO J, 21: 221-32.

Leng, G., et al. (1982). "The supraoptic nucleus as an osmoreceptor." Neuroendocrinology 34(1): 75-82.

Li, M., C. Du, D. Zhu, M. Shen, L. Cui, and J. Wang. 2012. 'Daytime variations of tear osmolarity and tear meniscus volume', Eye Contact Lens, 38: 282-7.

Li, Y., K. Kuang, B. Yerxa, Q. Wen, H. Rosskothen, and J. Fischbarg. 2001. 'Rabbit conjunctival epithelium transports fluid, and P2Y2(2) receptor agonists stimulate $\mathrm{Cl}(-$ ) and fluid secretion', Am J Physiol Cell Physiol, 281: C595-602.

Liedtke, W., et al. (2000). "Vanilloid receptor-related osmotically activated channel (VROAC), a candidate vertebrate osmoreceptor." Cell 103(3): 525-535.

Lindeman, R. D., et al. (1985). "Longitudinal studies on the rate of decline in renal function with age." J Am Geriatr Soc 33(4): 278-285.

Madden, L. C., A. Tomlinson, and P. A. Simmons. 2013. 'Effect of humidity variations in a controlled environment chamber on tear evaporation after dry eye therapy', Eye Contact Lens, 39: 169-74. 
830

831

832

833

834

835

836

837

838

839

840

841

842

843

844

845

846

847

848

849

850

851

852

853

854

855

856

857

858

859

860

861

862

863

864

865

866

867

868

869

870

871

872

873

874

875

876

877

Manz, F., and A. Wentz. 2005. 'The importance of good hydration for the prevention of chronic diseases', Nutr Rev, 63: S2-5.

Martin, A. D., et al. (1994). "Adipose tissue density, estimated adipose lipid fraction and whole body adiposity in male cadavers." Int J Obes Relat Metab Disord 18(2): 79-83.

Matz, R. (1996). "Dehydration in older adults." JAMA 275(12): 911-912.

McDonald, J. E., and S. Brubaker. 1971. 'Meniscus-induced thinning of tear films', Am J Ophthalmol, 72: 139-46.

Mentes, J. (2006). "Oral hydration in older adults: greater awareness is needed in preventing, recognizing, and treating dehydration." Am J Nurs 106(6): 40-49

Miller, K. L., K. A. Polse, and C. J. Radke. 2002. 'Black-line formation and the "perched" human tear film', Curr Eye Res, 25: 155-62.

Mircheff, A. K. 1989. 'Lacrimal fluid and electrolyte secretion: a review', Curr Eye Res, 8: 607-17.

Mishima, S. (1965). "Some Physiological Aspects of the Precorneal Tear Film." Arch Ophthalmol 73: 233-241.

Mishima, S., and D. M. Maurice. 1961. 'The oily layer of the tear film and evaporation from the corneal surface', Exp Eye Res, 1: 39-45.

Morgan, A. L., et al. (2002). "Age effects on body fluid distribution during exercise in the heat." Aviat Space Environ Med 73(8): 750-757.

Morley, J. (2000). "Water, water everywhere and not a drop to drink." J Gerontol A Biol Sci Med Sci 55(7): M359-360.

Moss, S. E., R. Klein, and B. E. Klein. 2008. 'Long-term incidence of dry eye in an older population', Optom Vis Sci, 85: 668-74.

Murube, J. 2009. 'Basal, reflex, and psycho-emotional tears', Ocul Surf, 7: 60-6.

Nelson, J. D. 1989. 'Managing the dry eye. Accurate diagnosis is the key', Postgrad Med, 85: $38-41,45-8,55$.

Nelson, J. D., and J. C. Wright. 1986. 'Tear film osmolality determination: an evaluation of potential errors in measurement', Curr Eye Res, 5: 677-81.

Niimi, J., B. Tan, J. Chang, Y. Zhou, A. Ghanekar, M. Wong, A. Lee, and M. C. Lin. 2013. 'Diurnal Pattern of Tear Osmolarity and Its Relationship to Corneal Thickness and Deswelling', Cornea, 32: 1305-10.

Oei, E., K. Paudel, A. Visser, H. Finney, and S. L. Fan. 2016. 'Is overhydration in peritoneal dialysis patients associated with cardiac mortality that might be reversible?', World $J$ Nephrol, 5: 448-54.

Olde Rikkert, M. G., et al. (1997). "Validation of multi-frequency bioelectrical impedance analysis in detecting changes in fluid balance of geriatric patients." J Am Geriatr Soc 45(11): 1345-1351.

Panel on Dietary Reference Intakes for Electrolytes, Water. Dietary reference intakes for water, potassium, sodium, chloride, and sulfate. Washington DC, USA: National Academies Press; 2004. www.nal.usda.gov/fnic/DRI/

DRI 'Water/water ' full'report.pdf. Washington DC, USA: National Academies Press, (accessed 14 April 2015).

Peng, C. C., C. Cerretani, R. J. Braun, and C. J. Radke. 2014. 'Evaporation-driven instability of the precorneal tear film', Advances in colloid and interface science, 206: 250-64.

Rikkert, M. G. M. O., et al. (1998). Age-related changes in body fluid compartments and the assessment of dehydration in older age. Hydration and Aging. M. Arnaud, Vellas, B.J., Albarede, J.L., Garry, P.J. eds. New York, NY Springer Publishing Company: 13-32. 
878

879

880

881

882

883

884

885

886

887

888

889

890

891

892

893

894

895

896

897

898

899

900

901

902

903

904

905

906

907

908

909

910

911

912

913

914

915

916

917

918

919

920

921

922

923

924

925

926

927

Rismondo, V., T. B. Osgood, P. Leering, M. G. Hattenhauer, J. L. Ubels, and H. F. Edelhauser. 1989. 'Electrolyte composition of lacrimal gland fluid and tears of normal and vitamin A-deficient rabbits', CLAO J, 15: 222-8.

Rolls, B. J. and P. A. Phillips (1990). "Aging and disturbances of thirst and fluid balance." Nutr Rev 48(3): 137-144.

Rose, G. E., and J. E. Wright. 1992. 'Pleomorphic adenoma of the lacrimal gland', $B r J$ Ophthalmol, 76: 395-400.

Sack, R. A., A. Beaton, S. Sathe, C. Morris, M. Willcox, and B. Bogart. 2000. 'Towards a closed eye model of the pre-ocular tear layer', Prog Retin Eye Res, 19: 649-68.

Sands, J. M. (2012). "Urine concentrating and diluting ability during aging." J Gerontol A Biol Sci Med Sci 67(12): 1352-1357.

Sen, D. K. and G. S. Sarin (1980). "Tear glucose levels in normal people and in diabetic patients." Br J Ophthalmol 64(9): 693-695.

Shiue, M. H., A. A. Kulkarni, H. J. Gukasyan, J. B. Swisher, K. J. Kim, and V. H. Lee. 2000. 'Pharmacological modulation of fluid secretion in the pigmented rabbit conjunctiva', Life Sci, 66: PL105-11.

Sollanek, K. J., et al. (2012). "Assessment of thermal dehydration using the human eye: What is the potential?" Journal of Thermal Biology 37: 111-117.

Stevenson, W., S. Pugazhendhi, and M. Wang. 2016. 'Is the main lacrimal gland indispensable? Contributions of the corneal and conjunctival epithelia', Surv Ophthalmol, 61: 616-27.

Stookey, J.D., High prevalence of plasma hypertonicity among community-dwelling older adults: results from NHANES III, J. Am. Diet. Assoc. 105 (2005) 1231-1239.

Sullivan, B.D., D. Whitmer, K.K. Nichols, A. Tomlinson, G.N. Foulks, G. Geerling, J.S. Pepose, V. Kosheleff, A. Porreco, M.A. Lemp, An objective approach to dry eye disease severity, Invest. Ophthalmol. Vis. Sci. 51 (2010) 6125-6130. doi:10.1167/iovs. 10-5390.

Taiara, C., and B. Smith. 1973. 'Palpebral dacryoadenectomy', Am J Ophthalmol, 75: 461-5.

Terry, J. E., and R. M. Hill. 1978. 'Human tear osmotic pressure: diurnal variations and the closed eye', Arch Ophthalmol, 96: 120-2.

Thaysen, J. H., and N. A. Thorn. 1954. 'Excretion of urea, sodium, potassium and chloride in human tears', Am J Physiol, 178: 160-4.

Thomas, D. R., et al. (2008). "Understanding clinical dehydration and its treatment." J Am Med Dir Assoc 9(5): 292-301.

Tomlinson, A., M. G. Doane, and A. McFadyen. 2009. 'Inputs and outputs of the lacrimal system: review of production and evaporative loss', Ocul Surf, 7: 186-98.

Tomlinson, A., S. Khanal, K. Ramaesh, C. Diaper, and A. McFadyen. 2006. 'Tear film osmolarity: determination of a referent for dry eye diagnosis', Invest Ophthalmol Vis Sci, 47: 4309-15.

Tomlinson, A., L. Madden, and E.I. Pearce. 2011. 'author reply to "Influence of modest changes in whole-body hydration on tear fluid osmolarity: important considerations for dry eye disease detection." ', Cornea, 30: 1517-18.

Tsubota, K. 1998. 'Tear dynamics and dry eye', Prog Retin Eye Res, 17: 565-96.

Tsubota, K., and K. Nakamori. 1995. 'Effects of ocular surface area and blink rate on tear dynamics', Arch Ophthalmol, 113: 155-8.

Tsubota, K., M. Yamada, and K. Urayama. 1994. 'Spectacle side panels and moist inserts for the treatment of dry-eye patients', Cornea, 13: 197-201.

Turpie, B., T. Yoshimura, A. Gulati, J. D. Rios, D. A. Dartt, and S. Masli. 2009. 'Sjogren's syndrome-like ocular surface disease in thrombospondin-1 deficient mice', Am. J. Pathol., 175: 1136-47. 
928

929

930

931

932

933

934

935

936

937

938

939

940

941

942

943

944

945

946

947

948

949

950

951

952

953

954

955

956

957

958

959

960

961

962

963

964

965

966

967

968

969

970

971

972

973

974

975
Ubels, J. L., K. K. Williams, D. Lopez Bernal, and H. F. Edelhauser. 1994. 'Evaluation of effects of a physiologic artificial tear on the corneal epithelial barrier: electrical resistance and carboxyfluorescein permeability', Adv Exp Med Biol, 350: 441-52.

Ubels, J. L., et al. (2006). "Gene expression in rat lacrimal gland duct cells collected using laser capture microdissection: evidence for K+ secretion by duct cells." Invest Ophthalmol Vis Sci 47(5): 1876-1885.

Uchino, M., M. Dogru, Y. Yagi, E. Goto, M. Tomita, T. Kon, M. Saiki, Y. Matsumoto, Y. Uchino, N. Yokoi, S. Kinoshita, and K. Tsubota. 2006. 'The features of dry eye disease in a Japanese elderly population', Optom Vis Sci, 83: 797-802.

Ungaro, C. T., et al. (2015). "Non-invasive estimation of hydration status changes through tear fluid osmolarity during exercise and post-exercise rehydration." Eur J Appl Physiol 115(5): 1165-1175.

Valim, V., V. F. Trevisani, J. M. de Sousa, V. S. Vilela, and R. Belfort, Jr. 2015. 'Current Approach to Dry Eye Disease', Clin Rev Allergy Immunol, 49: 288-97.

Van Haeringen, N. J. 1981. 'Clinical biochemistry of tears', Surv Ophthalmol, 26: 84-96.

Walsh, N. P., M. B. Fortes, and M. Esmaeelpour. 2011. 'Influence of modest changes in whole-body hydration on tear fluid osmolarity: important considerations for dry eye disease detection', Cornea, 30: 1517; author reply 17-8.

Walsh, N. P., M. B. Fortes, P. Raymond-Barker, C. Bishop, J. Owen, E. Tye, M. Esmaeelpour, C. Purslow, and S. Elghenzai. 2012. 'Is whole-body hydration an important consideration in dry eye?', Invest Ophthalmol Vis Sci, 53: 6622-7.

Watsky, M. A., M. M. Jablonski, and H. F. Edelhauser. 1988. 'Comparison of conjunctival and corneal surface areas in rabbit and human', Curr Eye Res, 7: 483-6.

Welch, C., and J. Baum. 1988. 'Tarsorrhaphy for corneal disease in patients with rheumatoid arthritis', Ophthalmic Surg, 19: 31-2.

Willshire, C., et al. (2017). "Estimating basal tear osmolarity in normal and dry eye subjects." Contact Lens and Anterior Eye. 2017 Sep 22. pii: S1367-0484(17)30179-0. doi: 10.1016/j.clae.2017.09.005. [Epub ahead of print]

Wolff, A., et al. (2015). "Are patients admitted to hospitals from care homes dehydrated? A retrospective analysis of hypernatraemia and in-hospital mortality." J R Soc Med 108(7): 259-265.

Xiao, H., J. Barber, and E. S. Campbell. 2004. 'Economic burden of dehydration among hospitalized elderly patients', Am J Health Syst Pharm, 61: 2534-40.

Yokoi, N., A. J. Bron, and G. A. Georgiev. 2014. 'The precorneal tear film as a fluid shell: the effect of blinking and saccades on tear film distribution and dynamics', Ocul Surf, 12: 252-66.

Yokoi, N., A. Komuro, K. Nishida, and S. Kinoshita. 1997. 'Effectiveness of hyaluronan on corneal epithelial barrier function in dry eye', Br J Ophthalmol, 81: 533-6.

Yoshimura, H., and K. Hosokawa. 1963. 'Studies on the mechanism of salt and water secretion from the lacrimal gland', Jpn J Physiol, 13: 303-18.

Zerbe, R. L., et al. (1991). "The reproducibility and heritability of individual differences in osmoregulatory function in normal human subjects." J Lab Clin Med 117(1): 51-59.

Zhu, H. and A. Chauhan (2007). "Tear dynamics model." Curr Eye Res 32(3): 177-197.

Zizza, C. A., et al. (2009). "Total water intakes of community-living middle-old and oldestold adults." J Gerontol A Biol Sci Med Sci 64(4): 481-486. 